\title{
TIPOLOGÍA, CONSERVACIÓN Y GESTIÓN DE LAS ZONAS HÚMEDAS DEL SURESTE ESPAÑOL
}

\author{
F. Robledano*, J. F. Calvo*, M. A. Esteve*, J. A. Palazón*, L. Ramírez* y J. Mas** \\ * Departamentode Biología Animal y EcologiA. Facultad de Biología, Universidad de Murcia, Murcia \\ ** InstitutoEspañol de Oceanografía.CentroOceanográfico del Mar Menor, Lo Pagan (Murcia)
}

Palabras clave: Wetlands, tipology, conservation, management, SE Spain.

\begin{abstract}
TIPOLOGY,CONSERVATION AND MANAGEMENT OFTHE WETLANDSOFSOUTHEASTSPAIN

A study has been carried out in 17 wetlands of Alicante and Murcia (SESpain), in order toestablish a tipology of these areas.

Information on ten physical and biotic parameters has been collected for each wetland site, and multivariate statistics(clusterand factor analysis), combined tobuild up a hyerarchical ordination, havebeen used todefine 9 types of wetlands (Salt fields in use, Mar Menor, La Pedrera Reservoir, Encañizadas, Salt fields lagoons, "El Hondo" Reservoirs, Non-Saline lagoons, Rambla de las Moreras and abandoned Salt fields).These have been lately characterized by their vertebrate fauna (mainly waterfowl) and some conservation and management topics (ownership, current use, threats and management proposals).

The rclative homogeneity of some types (particularlythe "Salt fieldsin use" and "abandoned salt fields"), is concluded, without that meaning a faunistic homogeneity. The absence of guarenteesfor the conservation of most sites is emphasized, and alternative management proposalssuggested.
\end{abstract}

\section{INTRODUCCIÓN}

Enel marco del reciente reconocimiento internacional de los valores que albergan las zonas húmedas (Fuentes, 1982), surgen iniciativas, a escala regional, para la investigación de estos sistemas. En muchos casos elénfasis se pone en la valoración de los mismos con respecto a determinados grupos biológicos (Amatet al., 1985; Ballarín, 1985), pero algunos autores han señalado la necesidad de acudir a criterios más amplios para evitar la infravaloración de determinados encla-

Limnetica, 3 (2):311-320 (1987)

(c) Asociación Española de Limnología, Madrid, Spain ves (Amat, 1982; Arnat et al., 1985). De lo anterior se desprende la necesidad delestablecimiento de una tipología regional, basada e n diversos criterios, como paso previo fundamental para la correcta gestión d e las zonas húmedas.

Los humedales del litoral del sureste español abarcan, dentro de un origen similar para la mayoría d e ellos (Lillo, 1979), una amplia panorámica de situaciones ambientales, fruto de una intensa utilización y transformación por parte del hombre. El conocimiento riguroso de estas situaciones es básico a la hora de establecer los objetivos y prioridades de una política de gestión. 
Enresumen, losobjetivos del presenteestudio se pueden concretar en:

- Tipificar, con una metodología sencilla, y en base a información fácilmente asequible (ver Morgan \& Boy, 1982), los distintos humedales costeros del sureste español (provincias de Alicante y Murcia).

- Caracterizar los tipos resultantes en función de su fauna de vertebrados y de los aspectos relacionados con su conservación y gestión.

\section{ÁREA DE ESTUDIO}

Comprende diecisiete medios inundados de las provincias de Alicante y Murcia (Fig. 1). De entre las localidades seleccionadas, la mayoría tienen un origen predominantemente marino $(1$, $7,8,9,10,11,12,13,14,15,16)$ o marino-fluvial $(2$, $3,4,5,17)$, y sólo una (6) es de reciente creación artificial. Muchas de ellas han pasado o se encuentran actualmente en fase de aprovechamiento salinero $(1,4,7,8,9,11,13,14,15,16)$, y dos $(5$, 6) almacenan agua para riegos. Para una más amplia información sobre estas zonas, pueden consultarse, entre otros, los trabajos de Egea y Esteva (1981), Navarroy Navarro (1982), Colegio Oficialde Arquitectosde Valencia (1984) y Calvo e Iborra (1986).

\section{METODOLOGIA}

Para cada localidad estudiada se recopiló información relativa a diez variables del medio físico y biótico.

1.- Superficie de la cubeta.

2.-índicedecomplejidad morfológica.Se utilizóel propuesto por Tuite efal. (1984), que relaciona la longitud de orilla (l)en kilómetros, y la superficie de la cubeta (S) en hectáreas, por medio de la expresión:

$$
I=100 \mathrm{~L} / 4 \mathrm{~S}
$$

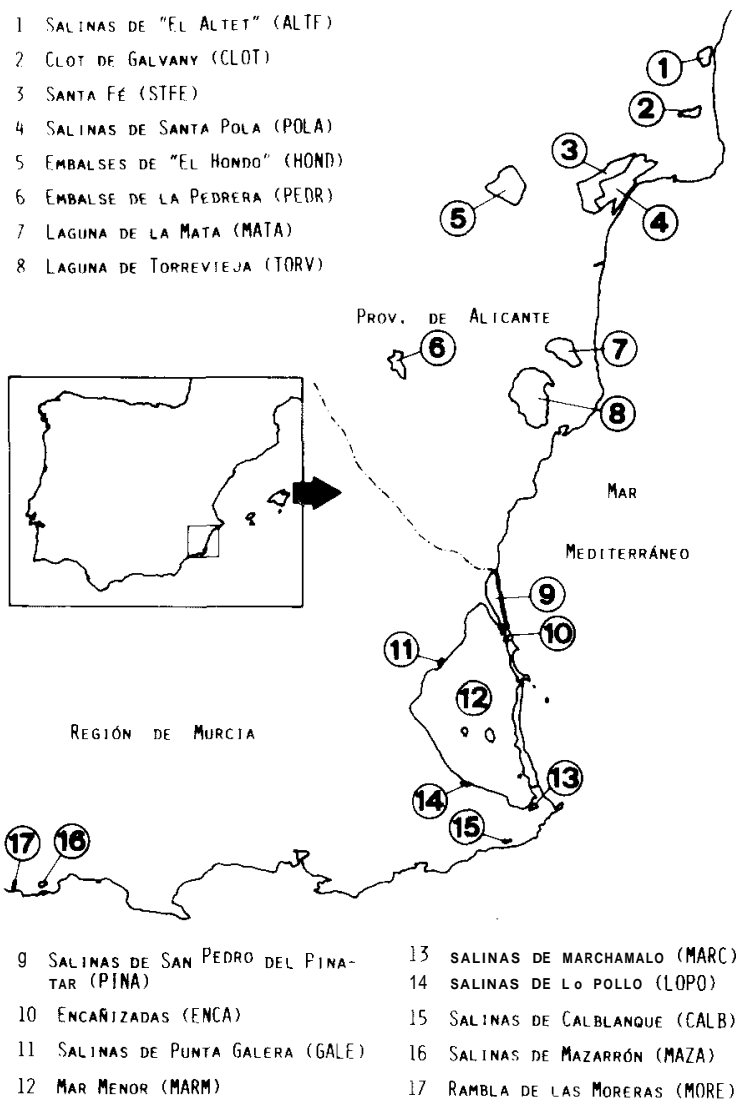

Figura1.-Área de estudio Study area.

\section{3.- Profundidad media}

4.- Profundidad máxima

(Paraambas profundidades(varialbes 3 y 4 )se consideraron cuatro rangos:

$$
\begin{aligned}
& 1=<10 \mathrm{~cm} \\
& 2=11-50 \mathrm{~cm} \\
& 3=50 \mathrm{~cm}-2 \mathrm{~m} \\
& 4=>2 \mathrm{~m}
\end{aligned}
$$

5.- Fluctuación del nivel del agua, expresada por la relación:

$$
\frac{\mathrm{N} \max -\mathrm{N} \min }{\mathrm{N} \min } \times 100
$$

6.- Contribución estimada (en \%) de la precipifaciónal volumen mediode agua almacenado. 
7.- Salinidad media del agua en gramos/litro. Se han considerado cuatro rangos de salinidad (ver Kiener, 1978):

$$
\begin{aligned}
& 1=<10 \mathrm{~g} / 1 \text { (oligo-mesohalina) } \\
& 2=11-37 \text { (meso-euhalina) } \\
& \mathbf{3}=38-50 \text { (hipersalina) } \\
& 4=>50 \text { (" ) }
\end{aligned}
$$

8,9 y 10.-Porcentaje desuperficiecubierta pordistintos tipos devegetación ribereña, con respectoal total cubierto por ésta. Los tipos consideradoshan sido: (8)carrizal,(9)saladar-juncaly (10) vegetaciónde arenales.

Esta informaciónfue obtenidaa partirde fotogramasaéreos(escala1:18.000; vuelodel Ministerio de Agricultura, 1977), cartografía (1:5.000, $1: 25.000$ y $1: 50.000$ ), y del conocimiento previo (bibliográficœ inédito)existente, siendocompletado con la inspección directa rápida de algunas localidades, según la metodología propuesta por Morgan \& Boy (1982). Ésta se almacenó en una ficha para cada localidad, a la que se añadió información relativa a su fauna de vertebradosy a los aspectos relacionadoscon su conservación y gestión.

La matriz de 17 casos $x 10$ variables resultante fue sometida a dos tipos de análisis multivariantes, "cluster" y "factor analysis" (BMDP2M y
BMDP4M;Dixon \& Brown,1982).Los tiposresultantes de la superposición de ambos análisis (ordenación jerárquica) fueron posteriormente caracterizados, de forma genérica, en cuanto a su fauna de vertebrados (avifauna, fundamentalmente), y a cuatro aspectos relacionados con su conservación y gestión (situación legal, amenazas, usos actuales y propuestas de gestión).Estos aspectos han sido tipificados en la tabla I.

\section{RESULTADOS Y DISCUSIÓN}

En la tabla II se recogen los valoresde las diez variables consideradas para las diecisiete localidades estudiadas. Lasfiguras2,3 y 4 y la tabla III, recogen los resultadosde los análisisde "cluster" y "factor analysis", respectivamente. Además la figura resume los de la ordenación jerárquica resultantede superponer losdosanálisismencionados. En la figura 2 se aprecia la agrupación bastante coherente de algunas localidades. Las salinas abandonadas (ALTE, LOPO, MAZA Y GALE) Se separan del restode localidades, lo mismoque el Mar Menor (MARM) y la Rambla de las Moreras (MORE).Dentro del grupo constituido por las demás, el Embalse de la Pedrera (PEDR) y las salinas

\begin{tabular}{|c|c|c|c|}
\hline SITUACION LEGAL: & USOS: & AMENAZAS: & PROPUESTAS DEGESTIÓN: \\
\hline $\begin{array}{l}\text { 1. Espacionatural protegido } \\
\text { 2. Zona no urbanizable } \\
\text { 3. Zonaurbanizable } \\
\text { 4. Propiedad pública/estatal } \\
\text { 5. Propiedad particular }\end{array}$ & $\begin{array}{l}\text { 1. Industrial } \\
\text { 2. Riegos } \\
\text { 3. Caza } \\
\text { 4. Pesca/acuicultura } \\
\text { 5. Urbanístico/turístico } \\
\text { 6. Agrícola } \\
\text { 7. No definido }\end{array}$ & $\begin{array}{l}\text { 1. Peligro de desaparición } \\
\text { abandono de actividades } \\
\text { tradicionales } \\
\text { 2. Presiónurbanística } \\
\text { 3. Contaminación/vertidos } \\
\text { 4. Presióncinegética } \\
\text { 5. Uso recreativo intenso } \\
\text { 6. Grandes obras de } \\
\text { infraestructura } \\
\text { 7. Destrucción de la } \\
\text { vegetación } \\
\text { 8. Noconocidas }\end{array}$ & $\begin{array}{l}\text { 1. Recuperación del nivelde agua y /o } \\
\text { de las actividades tracionales } \\
\text { 2. Declaracióncomozona nourbanizable } \\
\text { y evitaciónde grandes obras de } \\
\text { infraestructura } \\
\text { 3. Depuración aguas/cese de vertidos } \\
\text { 4. Control de la caza/pesca } \\
\text { 5. Control uso recreativo } \\
\text { 6. Aprovechamientodidácticoy científi- } \\
\text { co } \\
\text { 7. Declaracióncomoespacionatural pro- } \\
\text { tegidoo mejoradelestatutode protec- } \\
\text { ción } \\
\text { 8. Cesede ladestmcciónde la vegetación } \\
\text { y recuperaciónde ésta } \\
\text { 9. Ordenaciónintegraldel territorio }\end{array}$ \\
\hline
\end{tabular}

Tabla I: Clave numérica correspondiente a los aspectos relacionadoscon la conservación y gestión de las localidades estudiadas.

CLAVE: 


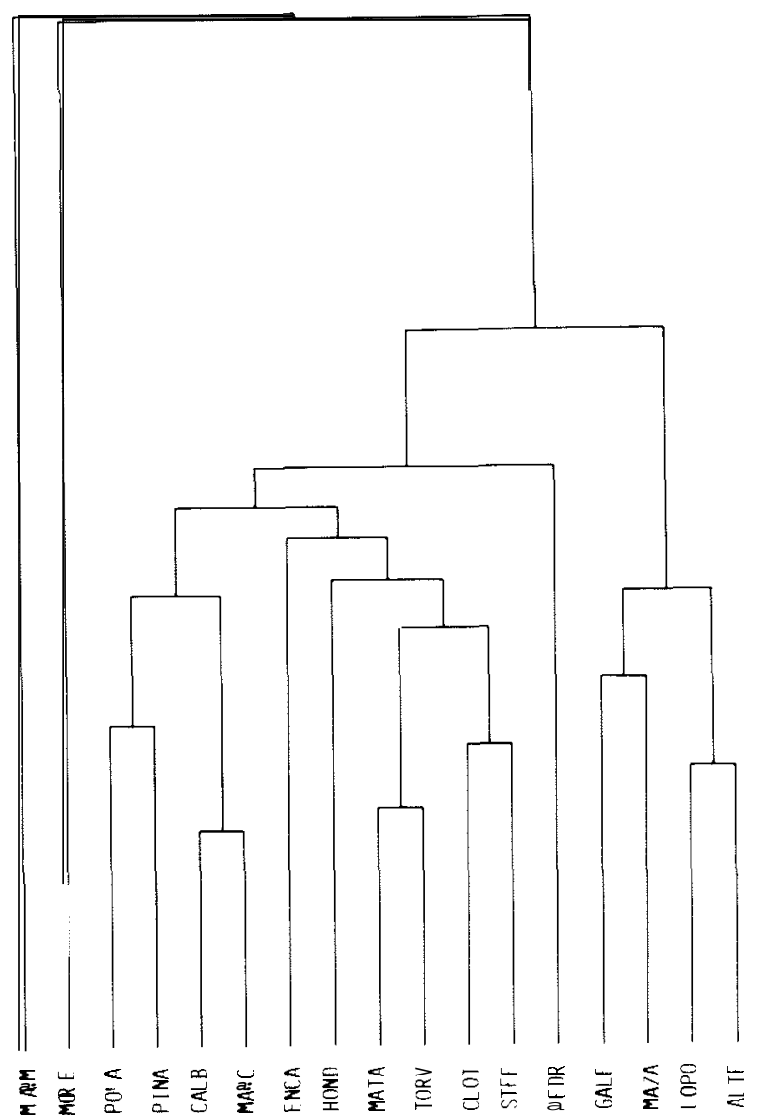

Figura 2 -Resultados del análisis de "cluster" de la matriz de 17 casos $x 10$ variables

Results of the cluster analysis of the data matrix en funcionamiento (POLA, PINA, CALB, MARC) se separan de un grupo constituido por las zonas de morfología más lagunar (ENCA, HOND, MATA, TORV, CLor, $\mathbf{m}$ ) Dentro de este último, las salinas de morfología lagunar (MATA, TORV) aparecen agrupadas y separadas de las lagunas (CLOT, STFE) "dulces" (meso- o polihalinas).

La figura 3 refuerza y ayuda a interpretar las agrupacionesanteriores. El eje Idel "factor analysis", en primer lugar, separa a las zonas de mayor profundidad media y máxima, y mayor superficie (MARM, HOND), de las de mayor complejidad estructural (salinas, en general). El eje II opone mayor fluctuación y contribución de la precipitación al volumen medio de agua, a mayor salinidad media, apareciendo las salinas en uso claramente en la parte negativa del eje, lo mismo que las encañizadas del mar Menor (ENCA), y las salinas de morfología lagunar (MATA, TORV), si bien estas últimas con un componente de fluctuación que lasacerca más al origende coordenadas. En el lado opuesto aparecen las salinas abandonadas (porsu mayor fluctuación y porestar alimentadas casi exclusivamente por agua de lluvia), y las "lagunas" y charcas dulces o salobres más fluctuantes (HOND, CLOT, MORE). El eje III opone mayor superficie de vegetación de arenales a mayor superficie cubierta por carrizal, separando claramente a la mayoría de las salinas, por su condi-

Tabla II: Valores de las diez variables físicas y bióticas especificadas en "Métodos" para cada una de las localidades estudiadas.

\begin{tabular}{|c|c|c|c|c|c|c|c|c|c|c|}
\hline & $\underline{1}$ & $\underline{2}$ & 3 & 4 & $\underline{5}$ & 6 & 7 & 8 & 9 & 10 \\
\hline 1. EL ALTET & 152 & 7,65 & 1 & 2 & 100 & 95 & 1 & 52 & 38 & ${ }_{10}^{10}$ \\
\hline 2. CLOTGALVANY & 35 & 4,28 & 2 & 3 & 70 & 50 & 2 & 55 & 45 & 0 \\
\hline 3. STA FE & 216 & 2,71 & 2 & 3 & 10 & 30 & 1 & 55 & 45 & 0 \\
\hline 4. STA POLA & 1114 & 2,51 & 2 & 3 & 10 & 10 & 4 & 14 & 49 & 7 \\
\hline 5. EL HONDO & 2453 & 0,72 & 2 & 3 & 25 & 90 & 1 & 28 & 72 & 0 \\
\hline 6. LA PEDRERA & 650 & 0,77 & 3 & 3 & 0 & 15 & 1 & 0 & 0 & 0 \\
\hline 7. LA MATA & 602 & 0,44 & 3 & 3 & 20 & 33 & 4 & 40 & 60 & 0 \\
\hline 8. TORREVIEJA & 1463 & 0,30 & 2 & 3 & 10 & 50 & 4 & 35 & 65 & 0 \\
\hline 9. PINATAR & 533 & 5,20 & 2 & 3 & 0 & 5 & 3 & 9 & 27 & 64 \\
\hline 10. ENCAÑIZADAS & 164 & 1,92 & 2 & 3 & 0 & 0 & 3 & 0 & 100 & 0 \\
\hline 11. PUNTA GALERA & 18 & 13,05 & 1 & 1 & 100 & 100 & 1 & 0 & 72 & 28 \\
\hline 12. MARMENOR & 13000 & 0,12 & 4 & 4 & 0 & 0 & 3 & 2 & 42 & 56 \\
\hline 13. MARCHAMALO & 55 & 10,45 & 2 & 2 & 0 & 0 & 4 & 0 & 79 & 21 \\
\hline 14. LO POLLO & 16 & 11,05 & 1 & 1 & 100 & 100 & 1 & 36 & 44 & 20 \\
\hline 15. CALBLANQUE & 15 & 13,50 & 2 & 2 & 0 & 0 & 4 & 0 & 61 & 39 \\
\hline 16. MAZARRÓN & 38 & 12,5 & 1 & 2 & 100 & 100 & 1 & 0 & 100 & 0 \\
\hline 17. LASMORERAS & 1,5 & 16,6 & 3 & 3 & 75 & 40 & 1 & 75 & 25 & 0 \\
\hline
\end{tabular}




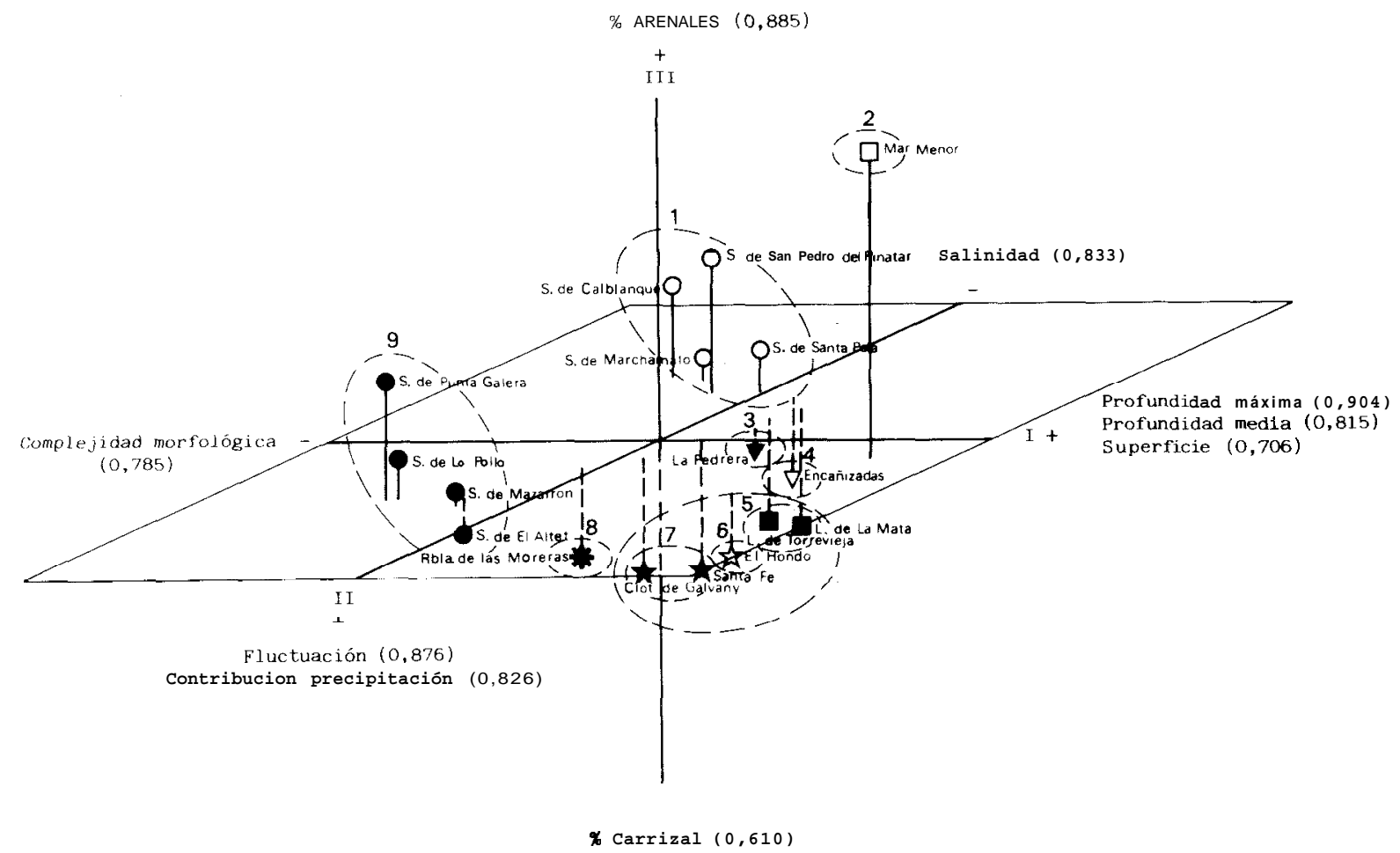

Figura3.- Representacióndelas localidades estudiadas en el espaciodefinidopor los tres primeros ejes del "factor analysis".Se han representado los tipos resultantesde la ordenaciónjerárquica(ver texto).

Representationofthe wetlandsstudied in the space defined by the axes I, II \& III of the factoranalysis. The types resulting from the hyerarchical ordination have been also represented (seetext).

Tabla III: Factores de carga del "factor analysis" de la matriz de 17 casos $x 10$ variables. Los valores de $\pm 0,25$ han sido reemplazados por 0 .

\begin{tabular}{lcccc} 
Variables & Factor $\mathbf{1}$ & Factor $\mathbf{2}$ & Factor $\mathbf{3}$ & Factor 4 \\
\hline Profundidad máxima & 0,904 & 0,000 & 0,000 & 0,000 \\
Profundidad media & 0,815 & $-0,261$ & 0,000 & $-0,296$ \\
Morfología (I. complejidad) & $-0,785$ & 0,253 & 0,000 & 0,000 \\
Superficie & 0,706 & 0,000 & 0,638 & 0,000 \\
Fluctuación & $-0,265$ & 0,876 & 0,000 & 0,000 \\
Salinidad media & 0,000 & $-0,833$ & 0,000 & 0,000 \\
Contribución precipitación & $-0,481$ & 0,826 & 0,000 & 0,000 \\
\% Arenales & 0,000 & $-0,283$ & 0,855 & 0,000 \\
\% Carrizal & 0,000 & 0,314 & $-0,610$ & $-0,543$ \\
\%Saladar & 0,000 & 0,000 & 0,000 & 0,936 \\
\hline
\end{tabular}


ción de lagunas litorales -más o menos transformadas- aisladas por barras de arena del mar originario (Lillo,1979y 1984), en la parte positiva del eje, de las lagunas, embalses y charcas,saladas o no, con importantes masas o cinturones decarrizo, en la parte negativa. Por último en la figura 4 se representan las localidades en el espacio definido por los factoresIII y IV determinados principalmente por el tipo de vegetación, donde se puede observar la distribución comentada para el eje III y que, en la parte positivadel eje IV se sitúan las localizadas que presentan mayores porcentajes de superficie cubierta por vegetación de saladares.

Los tipos resultantes de la ordenación jerárquica (Fig. 4), son los siguientes:

1. Salinas en funcionamiento: Sta. Pola (Pola), S. Pedro del Pinatar (PINA), Marchamalo (MARC), Calblanque (CALB).

AVIFAUNA:Tarroblanco(invemante, nidificante regular peroescaso); Flamenco(invernante, en paso y estival, con intentos de nidificación),larollimícolas(nidificantes),Zampullín cuellinegro (invernante y en paso), Gaviota de Audouin (invernante).

OTROS VERTEBRADOS: Apanius iberus

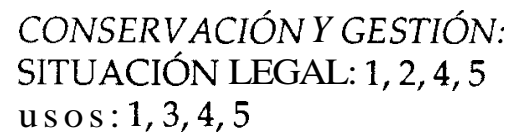

\section{Mar Menor (MARM)}

AVIFAUNA: Chorlitejopatinegro (nid.), Cormorán grande (inv.), Serreta mediana (inv.), Zampullín cuellinegro (inv.), Gaviota argéntea (nid.)

\section{CONSERVACIÓN Y GESTIÓN:}

$$
\text { S. } L .: 2,3,4,5
$$

USOS: $4,5,6$

AMENAZAS: $2,3,5,6,7$

PROPUESTAS DE GESTIÓN: 3, 5, 6, 9

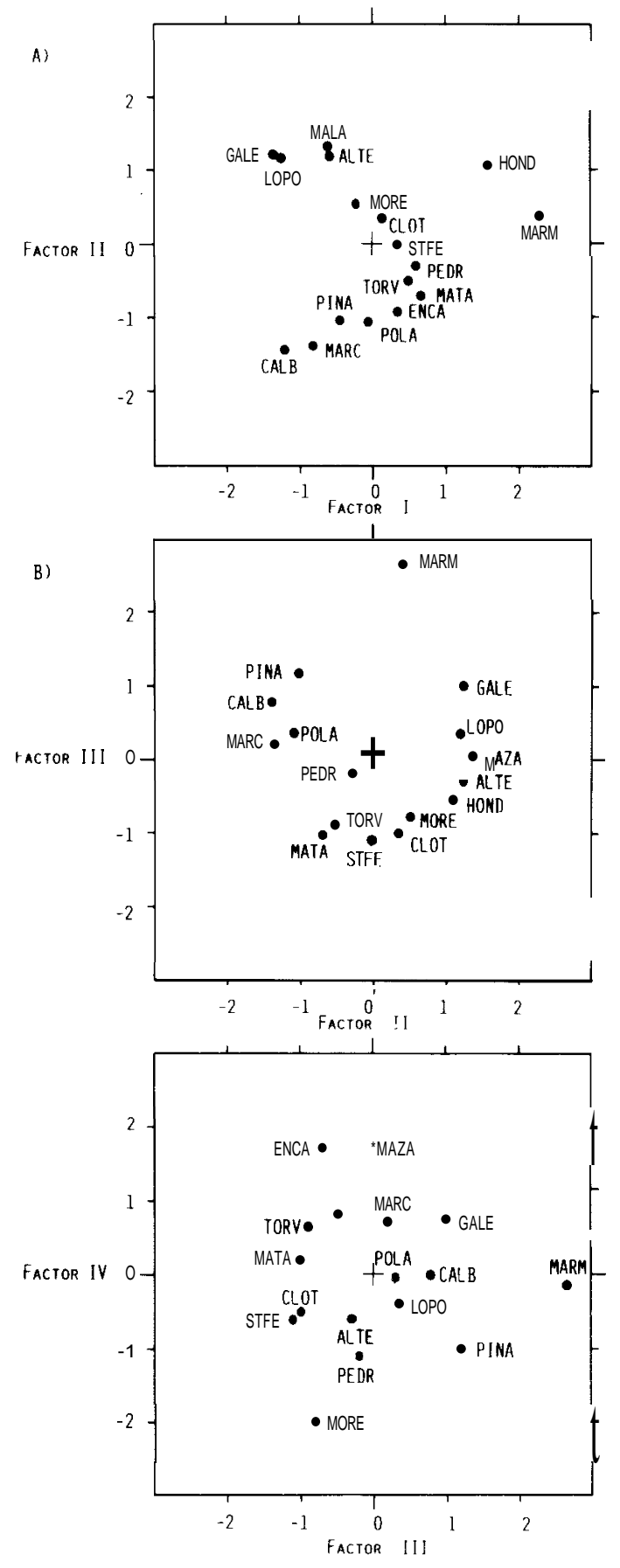

Figura 4.-Representaciónde las localidadesestudiadas en los planos definidos por los factores I y II (a), II y III y IV (c) del "factoranalysis".

Representationof the wetlandsstudied in the planesdefined by the factors I \& II (a), II \& III \& IV (c) of the factor analysis. 


\section{Embalse de la Pedrera (PEDR)}

AVIFAUNA: Anátidas (inv.), Focha común (inv., nid.), Somormujo lavanco (inv., nid.), Gaviota reidora (inv.).

CONSERVACIÓN Y GESTIÓN:

S.L.: 2,4

u s o s : 2

AMENAZAS: 8

PROPUESTAS DE GESTIÓN: -

4. Encañizadas (ENCA)

AVIFAUNA: Larolimícolas (nid., inv. y en paso), Ardeidas (inv.).

CONSERVACIÓN Y GESTIÓN:

S.L.: 1,4

USOS: 4

AMENAZAS: 5,6

PROPUESTASDE GESTIÓN: 2, 5, 6, 7

5. Salinas en lagunas: La Mata (MATA), Torrevieja (ToRv)

AVIFAUNA: Aguilucho cenizo (nid.) Tarro blanco (inv., nid. escaso pero regular), Larolimícolas (nid.), Flamenco (inv., en paso), Zampullín cuellinegro (inv., en paso), refugio de anátidas invernantes en localidades próximas (durante el período de caza).

CONSERVACIÓN Y GESTIÓN:

S.L.: 2,4

USOS: 1

AMENAZAS: 2, 3, 4, 5

PROPUESTASDE GESTIÓN: 3, 4, 5, 6, 7
6. Embalses de "El Hondo" (HoND)

AVIFAUNA: Anátidas (inv. nid.). Ardeidas (inv., nid.), Fumareles (nid.), Rálidos, pájaros del carnzal.

OTROS VERTEBRADOS: Aphanius iberus, Valencia hispanica (?)

CONSERVACIÓN Y GESTIÓN:

S.L.: 2,5

USOS: $2,3,4$

AMENAZAS: 3,4

PROPUESTAS DE GESTIÓN: 3, 4, 7

7. Lagunas no salinas: Clot de Galvany (CLor), Sta. Fe (STFE)

AVIFAUNA: Limícolas (inv.), Aguilucho cenizo (nid.) y lagunero (inv., nid.), Rálidos, pájaros del carnzal, Anátidas (inv. nid.), Ardeidas (inv. nid.), Fumareles (nid.).

\section{CONSERVACIÓN Y GESTIÓN:}

$$
\text { S.L.: } 2,3,5
$$

u s o $: 3,4$

AMENAZAS: $2,3,4,5$

PROPUESTASDE GESTIÓN: 3, 4, 5, 6, 7

8. Rambla de Las Moreras (MoRE)

AVIFAUNA: Rálidos, pájaros del carnzal.

CONSERVACIÓN Y GESTIÓN:

S.L.: 2,4

USOS: 7

AMENAZAS: $2,3,5$

PROPUESTAS DE GESTIÓN: 3,6 


\section{Salinas abandonadas (GAle, Lopo, Alte, MAZA)}

AVIFAUNA: Larolimícolas (nid.), Gaviotas (inv.).

CONSERVACIÓN Y GESTIÓN:

S.L.: $2,3,5$

u s o $\mathrm{s}: 5,7$

AMENAZAS: 1, 2, 3, 5, 7

PROPUESTASDE GESTIÓN: 1, 2, 3, 5, 6, 8, 9

Delos nueve tipos definidos, destaca la homogeneidad de los números 1 (SALINASEN FUNCIONAMIENTO) y 9 (SALINAS ABANDONADAS), independientemente de suextensióny otros factores.La definiciónde tipos, agrupando varias localidades, no conlleva una homogeneidad en cuanto a su fauna de vertebrados, particularmente en las agrupaciones más heterogéneas (p. ej.: LAGUNAS NO SALINAS). En otros casos, las diferencias faunísticas pueden deberse a un efecto de la superficie de la zona húmeda sobre el número de especies presentes (casode las SALINAS EN FUNCIONAMIENTO y, probablemente, de las SALINAS ABANDONADAS).

Considerando el elevado número de amenazas que pesan sobre la mayoría de las zonas estudiadas (particularmente sobre los tipos 1,2,5,

\section{BiBLIOGRAFía}

Амат, J. A. (1982):Ecología de las lagunas andaluzas, en: Las zonas húmedas en Andalucia. Monografías de la dirección general de Medio Ambiente. M.o.P.U. Madrid.

Amat, J. A.; Diaz Paniagua, C.; Herrera, C. M.; JordaNo, P.; OBESo, J. R. y SORIGUER, R. C. (1985):Criterios devaloración dezonashúmedasde importancianacional y regional en función de las aves acuáticas. Monografías, 35. ICONA. Madrid.

BaLlaRÍN, I. (1985): Clasificación de las zonas húmedas aragonesas de importancia internacional, nacional o regional en función de las aves acuáticas. Monografías, 40. ICONA. Madrid. 7y9, que abarcan 13 de las 17 localidades estudiadas), no pareceen absoluto garantizada la conservación de las mismas, máxime considerando la ineficacia de las medidas de protección existentes. Sólo 2 de las 17 localidades consideradas tienen estatutos específicos de protección (E.P.Y.P.S.A., 1985; Varios autors, 1987), y aún éstas experimentan importantes alteraciones en su interior o entorno.

Por ello, se considera imprescindible una actuación a cuatro niveles:

- Adecuada divulgación de los valoresnaturales de estas zonas.

- Declaración como espacios protegidos y revisiónde los estatutos de protecciónexistentes.

- Recuperación de zonas degradadas.

- Incorporación de estos medios a planes de ordenación integral del territorio con basesecológicas (ver, p. ej., Martin de Agar, 1984).

\section{AGRADECIMIENTOS}

Queremos expresar nuestro agradecimiento a J. A. Palazón, que llevóa caboel tratamiento dela información, y a V.Hernández Gil, por su colaboración en la redacción definitiva de este artículo.

Calvo, J. F. E Iborra, J. (1986): Estudio ecológico de la Laguna de la Mata. Instituto de Estudios Juan GilAlbert. Alicante.

COLEGIO OFICIAL DE ARQUITECTOS DE VALENCIA (1984): Espacios Naturales. Provincia de Alicante. Caja de Ahorros Provincial. Alicante.

Dixon, W. J. \& Brown, M. B. (eds.) (1982): BMDP Biomedical Computer Programs. University of California Press. Berkeley.

EgeA, J. M. y Esteve, M. A. (1981):Inventario, catalogación y delimitación de los Espacios Naturales de la Región Murciana. Diputación Provincialde Murcia. Murcia (inédito). 
E. P. Y. P. S. A. (1985):Plan Especial de Protección de Espacios Naturales de las Salinas de San Pedro, Coto de las Palomas y Playasde la Llana y del Mojón. Comunidad Autónoma de Murcia. Murcia.

FuENTEs, F. (1982): Calidad de vida, Medio Ambiente y Ordenación del Territorio.Textos Internacionales. Vol. III. CEOTMA-CIFCA. Madrid.

KIENER, A. (1978): Ecologie, physiologie et économie des eaux saumâtres. Masson. Paris.

LiLlo, M. J. (1979):Geomorfologia litoral del Mar Menor y del Bajo Segura. Tesis Doctoral. Universidad de Valencia.Valencia.

- (1984):"Consideraciones paleogeográficas sobre el endorreismo marginolitoral del sureste español (Provincias de Alicante y Murcia). Limnética, 1:8695.
Martin de Agar, P. (1984): Ecología y Planeamiento Territorial. Metodologia y estudio de casos en la Región Murciana. Tesis Doctoral. Universidad de Murcia. Murcia.

Morgan, N.C. \& BoY, V. (1982): "An ecological survey of standing waters in North West Africa: I. Rapid survey and classification". Biol. Conserv., 24: 5-44.

Navarro, J. D. y Navarro, J. (1982): "La avifauna de los Embalses de 'El Hondo'. Mediterránea Ser. Biol., 6:109-139.

Tutre, C. H.; Hanson, P. R. \& Owen, M. (1984): "Come ecologicalfactors affecting winter wildfowl distribution on inland waters in England and Wales, and the influence of water-based recreation". I. Appl. Ecol., 21:41-62.

$W$.AA.(1987): Plan Especial de Protecciónde Calblanque. Comunidad Autónoma de Murcia. Murcia. 